\title{
Betaxolol Hydrochloride
}

National Cancer Institute

\section{Source}

National Cancer Institute. Betaxolol Hydrochloride. NCI Thesaurus. Code C47413.

The hydrochloride salt form of betaxolol, a beta-1-selective adrenergic receptor antagonist without intrinsic sympathomimetic activity. Betaxolol hydrochloride acts on the heart and circulatory system and decreases cardiac contractility and rate, thereby reducing cardiac output. When applied topically in the eye, it lowers intraocular pressure by reducing aqueous humor secretion. 